\title{
Analytic definition of spin structure
}

Zhirayr Avetisyan, , Yan-Long Fang, , Nikolai Saveliev, and, and Dmitri Vassiliev

Citation: Journal of Mathematical Physics 58, 082301 (2017); doi: 10.1063/1.4995952

View online: http://dx.doi.org/10.1063/1.4995952

View Table of Contents: http://aip.scitation.org/toc/jmp/58/8

Published by the American Institute of Physics

\section{Articles you may be interested in}

A lower bound for the BCS functional with boundary conditions at infinity Journal of Mathematical Physics 58, 081901 (2017); 10.1063/1.4996580

Existence and mass concentration of pseudo-relativistic Hartree equation Journal of Mathematical Physics 58, 081501 (2017); 10.1063/1.4996576

Driving quantum systems with superoscillations Journal of Mathematical Physics 58, 082101 (2017); 10.1063/1.4996135

Existence and concentration of positive solutions for fractional nonlinear Schrödinger equation with critical growth

Journal of Mathematical Physics 58, 081502 (2017); 10.1063/1.4996578

Time-asymptotic interactions of two ensembles of Cucker-Smale flocking particles Journal of Mathematical Physics 58, 071509 (2017); 10.1063/1.4995607

Stability estimate for an inverse problem for the Schrödinger equation in a magnetic field with timedependent coefficient

Journal of Mathematical Physics 58, 071508 (2017); 10.1063/1.4995606

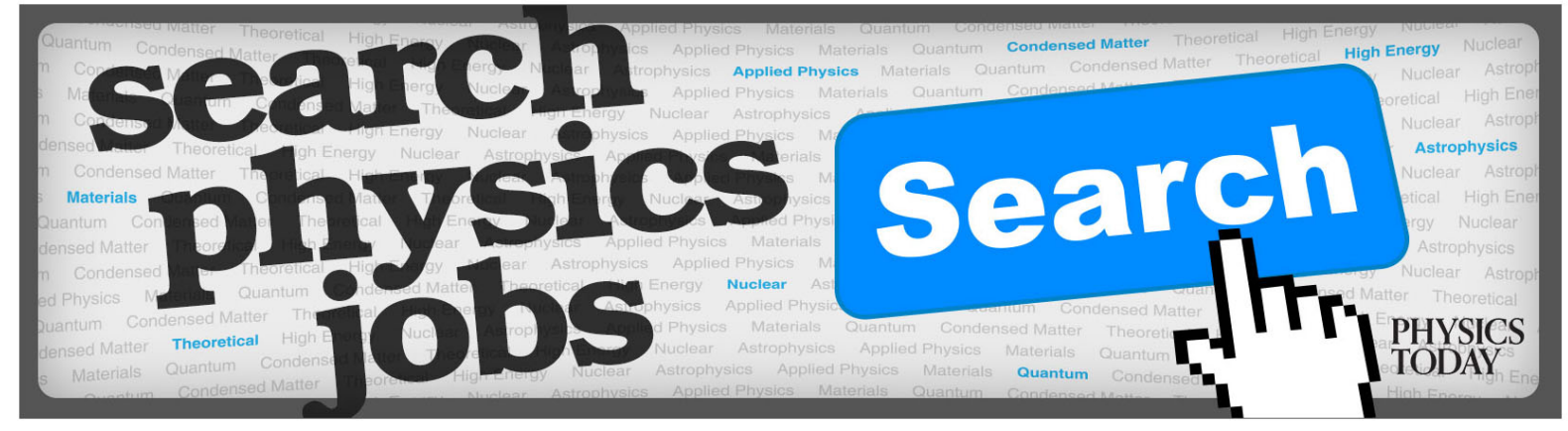




\title{
Analytic definition of spin structure
}

\author{
Zhirayr Avetisyan, ${ }^{1, a)}$ Yan-Long Fang, ${ }^{1, b)}$ Nikolai Saveliev, ${ }^{2, c)}$ \\ and Dmitri Vassiliev ${ }^{1, d)}$ \\ ${ }^{1}$ Department of Mathematics, University College London, Gower Street, London \\ WC1E 6BT, United Kingdom \\ ${ }^{2}$ Department of Mathematics, University of Miami, P.O. Box 249085, Coral Gables, \\ Florida 33124, USA
}

(Received 17 February 2017; accepted 11 July 2017; published online 2 August 2017)

\begin{abstract}
We work on a parallelizable time-orientable Lorentzian 4-manifold and prove that in this case, the notion of spin structure can be equivalently defined in a purely analytic fashion. Our analytic definition relies on the use of the concept of a nondegenerate two-by-two formally self-adjoint first order linear differential operator and gauge transformations of such operators. We also give an analytic definition of spin structure for the 3-dimensional Riemannian case. Published by AIP Publishing. [http://dx.doi.org/10.1063/1.4995952]
\end{abstract}

\section{PLAYING FIELD}

Let $M$ be a connected smooth 4-manifold without boundary, not necessarily compact.

In this paper, we will be working with functions on $M$, densities on $M$, vector fields on $M$, metrics on $M$, etc., and all of these will be assumed to be infinitely smooth. We will also be working with differential operators acting on $M$ and the coefficients of these differential operators will be assumed to be infinitely smooth.

We will use Latin letters for anholonomic (frame) indices and Greek letters for holonomic (tensor) indices. We will use the convention of summation over repeated indices; this will apply both to Greek and to Latin indices.

A half-density is a quantity $M \rightarrow \mathbb{C}$ which under changes of local coordinates transforms as the square root of a density. We will be working with compactly supported two-columns of half-densities and will define the inner product on pairs $v, w$ of such 2-columns as $\langle v, w\rangle:=\int_{M} w^{*} v d x$. Here $x=\left(x^{1}, x^{2}, x^{3}, x^{4}\right)$ are local coordinates on $M, d x=d x^{1} d x^{2} d x^{3} d x^{4}$, and the star stands for Hermitian conjugation.

Let $L$ be a first order linear differential operator acting on 2-columns of half-densities. In local coordinates, this operator reads $L=F^{\alpha}(x) \frac{\partial}{\partial x^{\alpha}}+G(x)$, where $F^{\alpha}(x)$ and $G(x)$ are some $2 \times 2$ matrixfunctions. The problem here is that these matrix-functions are not invariant under changes of local coordinates. The standard way of providing an invariant analytic description of the operator $L$ is by means of its principal and subprincipal symbols defined as

$$
\begin{gathered}
L_{\text {prin }}(x, p):=i F^{\alpha}(x) p_{\alpha}, \\
L_{\text {sub }}(x):=G(x)+\frac{i}{2}\left(L_{\text {prin }}\right)_{x^{\alpha} p_{\alpha}}(x)=G(x)-\frac{1}{2}\left(F^{\alpha}\right)_{x^{\alpha}}(x),
\end{gathered}
$$

respectively. Here $p=\left(p_{1}, p_{2}, p_{3}, p_{4}\right)$ is the dual variable (momentum) and the subscripts indicate partial derivatives. It is known that $L_{\text {prin }}$ and $L_{\text {sub }}$ are invariantly defined matrix-functions on $T^{*} M$ and $M$, respectively, see (Ref. 16, Subsection 2.1.3) and (Ref. 8, Appendix A) for details. Furthermore, it is

\footnotetext{
a)Email: z.avetisyan@ucl.ac.uk

b)Email: yan.fang.12@ucl.ac.uk

c) Email: saveliev@math.miami.edu

d) Email: d.vassiliev@ucl.ac.uk
} 
easy to see that the principal and subprincipal symbols uniquely determine our first order differential operator and that our operator is formally self-adjoint if and only if its principal and subprincipal symbols are Hermitian.

Further on we work with $2 \times 2$ formally self-adjoint first order differential operators.

Definition 1.1. We say that the operator $L$ is non-degenerate if

$$
L_{\text {prin }}(x, p) \neq 0, \quad \forall(x, p) \in T^{*} M \backslash\{0\} .
$$

Not every 4-manifold admits a non-degenerate operator. The following lemma establishes the appropriate criterion.

Lemma 1.2. The manifold $M$ admits a non-degenerate operator $L$ if and only if it is parallelizable.

Proof. Decomposing $L_{\text {prin }}(x, p)$ with respect to the standard basis

$$
s^{1}=\left(\begin{array}{ll}
0 & 1 \\
1 & 0
\end{array}\right), \quad s^{2}=\left(\begin{array}{rr}
0 & -i \\
i & 0
\end{array}\right), \quad s^{3}=\left(\begin{array}{rr}
1 & 0 \\
0 & -1
\end{array}\right), \quad s^{4}=\left(\begin{array}{ll}
1 & 0 \\
0 & 1
\end{array}\right)
$$

in the real vector space of $2 \times 2$ Hermitian matrices, we get

$$
L_{\text {prin }}(x, p)=s^{j} e_{j}^{\alpha}(x) p_{\alpha},
$$

where $e_{j}, j=1,2,3,4$, are some real-valued vector fields. Here each vector $e_{j}(x)$ has coordinate components $e_{j}^{\alpha}(x), \alpha=1,2,3,4$.

Formula (1.5) establishes a one-to-one correspondence between principal symbols and tetrads of vector fields. Furthermore, formula (1.5) allows us to rewrite the non-degeneracy condition (1.3) as

$$
\operatorname{det} e_{j}^{\alpha}(x) \neq 0, \quad \forall x \in M .
$$

But condition (1.6) is the condition of linear independence of the vector fields $e_{j}$.

Remark 1.3. The critical element of the above proof is the fact that the dimension of our manifold equals the dimension of the real vector space of $2 \times 2$ Hermitian matrices.

Remark 1.4. Further on, in Sec. IV, we will start referring to the above tetrad of vector fields as a framing. This will be a purely terminological change, for the benefit of readers accustomed to terminology used in topology and differential geometry.

Further on we assume that our 4-manifold $M$ is parallelizable.

\section{CORRESPONDENCE BETWEEN OPERATORS AND LORENTZIAN METRICS}

Observe that the determinant of the principal symbol is a quadratic form in momentum $p$,

$$
\operatorname{det} L_{\text {prin }}(x, p)=-g^{\alpha \beta}(x) p_{\alpha} p_{\beta} .
$$

We interpret the real coefficients $g^{\alpha \beta}(x)=g^{\beta \alpha}(x), \alpha, \beta=1,2,3,4$, appearing in formula (2.1) as components of a (contravariant) metric tensor. It is known (Ref. 9, Lemma 2.1) that this metric is Lorentzian, i.e., it has three positive eigenvalues and one negative eigenvalue.

It turns out that the Lorentzian metric defined in accordance with formula (2.1) has an additional geometric property. In order to describe this property, we need some definitions.

Definition 2.1. A vector field $u$ is said to be spacelike if $\left(g_{\alpha \beta} u^{\alpha} u^{\beta}\right)(x)>0, \forall x \in M$, lightlike if $\left(g_{\alpha \beta} u^{\alpha} u^{\beta}\right)(x)=0, \forall x \in M$, and timelike if $\left(g_{\alpha \beta} u^{\alpha} u^{\beta}\right)(x)<0, \forall x \in M$.

Definition 2.2. The Lorentzian manifold $(M, g)$ is said to be time-orientable if it admits a timelike vector field. 
Observe that the trace of the principal symbol is a linear form in momentum $p$ and the coefficients of this linear form can be interpreted as components of a vector field. With the standard choice of Pauli matrices (1.4), the linear form in question reads

$$
\operatorname{tr} L_{\text {prin }}(x, p)=2 e_{4}{ }^{\alpha} p_{\alpha} .
$$

It is easy to see that the vector field $e_{4}$ is timelike. Thus, formula (2.1) defines a time-orientable Lorentzian metric on our parallelizable 4-manifold $M$.

Let us now perform the above argument the other way round. Suppose we have a parallelizable 4-manifold $M$ equipped with a time-orientable Lorentzian metric. In this case, we have a timelike vector field which we will denote by $e_{4}$. Without loss of generality, we may assume that this timelike vector field is normalised, i.e., that $\left(g_{\alpha \beta} e_{4}{ }^{\alpha} e_{4}{ }^{\beta}\right)(x)=-1, \forall x \in M$.

Lemma 2.3. One can choose vector fields $e_{1}, e_{2}$, and $e_{3}$ such that the tetrad $e_{j}, j=1,2,3,4$, is linearly independent at all points of the manifold.

Proof. Let us fix a trivialization $T M=M \times \mathbb{R}^{4}$ and view $e_{4}$ as a smooth map $M \rightarrow S^{3}$. Since $S^{3}$ is parallelizable, its orthonormal frame bundle $S O(3) \rightarrow S O(4) \rightarrow S^{3}$ is trivial and therefore admits a section $S^{3} \rightarrow S O(4)$. Composing this section with the map $M \rightarrow S^{3}$, we obtain the desired tetrad.

Remark 2.4. The above argument also works for parallelizable manifolds of dimension 2 and 8 .

Applying now the Gram-Schmidt process, we obtain new vector fields $e_{1}, e_{2}, e_{3}$, and $e_{4}$ such that

$$
\left(g_{\alpha \beta} e_{j}^{\alpha} e_{k}^{\beta}\right)(x)=\left\{\begin{aligned}
0 & \text { if } j \neq k, \\
+1 & \text { if } j=k \neq 4, \\
-1 & \text { if } j=k=4,
\end{aligned}\right.
$$

for all $x \in M$. Here the Gram-Schmidt process works because the restriction of a Lorentzian metric to the orthogonal complement of a timelike vector gives a Riemannian metric. Finally, substituting our tetrad $e_{j}, j=1,2,3,4$, into (1.5), we obtain a principal symbol with the property (2.1).

\section{GAUGE TRANSFORMATIONS AND SPIN STRUCTURE}

From now on, the time-orientable Lorentzian metric is assumed to be fixed. We will work with all possible $2 \times 2$ formally self-adjoint non-degenerate first order linear differential operators corresponding, in the sense of formula (2.1), to the given metric. It was shown in Sec. II that the set of such operators is non-empty. Our aim is to classify operators corresponding to the given metric.

We specify an orientation on our manifold and define the topological charge of our operator as

$$
\mathbf{c}_{\text {top }}:=-\frac{i}{2} \sqrt{\left|\operatorname{det} g_{\alpha \beta}\right|} \operatorname{tr}\left(\left(L_{\text {prin }}\right)_{p_{1}}\left(L_{\text {prin }}\right)_{p_{2}}\left(L_{\text {prin }}\right)_{p_{3}}\left(L_{\text {prin }}\right)_{p_{4}}\right)=\operatorname{sgn} \operatorname{det} e_{j}^{\alpha},
$$

with the subscripts $p_{1}, p_{2}, p_{3}, p_{4}$ indicating partial derivatives with respect to the components of momentum $p=\left(p_{1}, p_{2}, p_{3}, p_{4}\right)$. It is easy to see that the number $\mathbf{c}_{\text {top }}$ defined by formula (3.1) can take only two values, +1 or -1 , and describes the orientation of the principal symbol relative to our chosen orientation of local coordinates $x=\left(x^{1}, x^{2}, x^{3}, x^{4}\right)$.

Let us choose a timelike co-vector field $q$ and use it as a reference. It is easy to see that the real-valued scalar function $\operatorname{tr} L_{\text {prin }}(x, q(x))$ does not vanish. We define the temporal charge of our operator as

$$
\mathbf{c}_{\mathrm{tem}}:=\operatorname{sgn} \operatorname{tr} L_{\mathrm{prin}}(x, q(x))=\operatorname{sgn}\left(q_{\alpha} e_{4}{ }^{\alpha}\right),
$$

see also formula (2.2). Note that $q_{\alpha} e_{4}{ }^{\alpha} \neq 0$ because both $q$ and $e_{4}$ are timelike. The number $\mathbf{c}_{\text {tem }}$ defined by formula (3.2) describes the orientation of the principal symbol relative to our chosen time orientation.

We perform a primary classification of our operators based on the values of their topological and temporal charges. Obviously, the four classes of operators in this primary classification correspond to the four connected components of the Lorentz group. 
Further on, we assume the topological and temporal charges to be fixed.

In order to classify our operators further, we introduce an arbitrary smooth $2 \times 2$ complex-valued matrix-function $R$ of determinant one,

$$
R: M \rightarrow S L(2, \mathbb{C})
$$

and consider the transformation

$$
L \mapsto R^{*} L R .
$$

Transformation (3.4) of our differential operator $L$ induces the transformation

$$
L_{\text {prin }} \mapsto R^{*} L_{\text {prin }} R
$$

of its principal symbol. (The induced transformation of the subprincipal symbol will be considered later in Sec. VI.) Formulae (2.1), (3.3), and (3.5) imply that transformation (3.4) preserves our Lorentzian metric $g$. Furthermore, it is easy to see that transformation (3.4) preserves the topological charge (3.1) and the temporal charge (3.2).

We interpret (3.4) as a gauge transformation because it preserves the prescribed metric as well as the prescribed topological and temporal charges.

Our analytic definition of spin structure is formulated as follows.

Definition 3.1. We say that the operators $L$ and $\tilde{L}$ are equivalent if

$$
\tilde{L}_{\text {prin }}=R^{*} L_{\text {prin }} R
$$

for some smooth matrix-function (3.3). An equivalence class of operators is called spin structure.

\section{MAIN RESULT}

In this section, we will show that our analytic definition of spin structure, Definition 3.1, is equivalent to the traditional geometric definition.

We begin by restating our analytic definition, Definition 3.1, in terms of framings. By a frame at a point $x \in M$, we mean a positively oriented and positively time-oriented orthonormal, in the Lorentzian sense (2.3), frame in the tangent space $T_{x} M$ and by a framing of $M$ a choice of a frame at every point $x \in M$ depending smoothly on the point. In our case, we have an explicit formula (1.5) establishing a one-to-one correspondence between principal symbols and framings. Any two framings of the same manifold $M$ are related by a uniquely defined smooth function $f: M \rightarrow S O^{+}(3,1)$, where $\mathrm{SO}^{+}(3,1)$ is the identity component of the Lorentz group. Rephrasing Definition 3.1, we will say that two framings are equivalent if the function $f$ relating them factors as

$$
f: M \rightarrow S L(2, \mathbb{C}) \stackrel{\mathrm{Ad}}{\longrightarrow} S O^{+}(3,1),
$$

where $\operatorname{Ad}: S L(2, \mathbb{C}) \rightarrow S O^{+}(3,1)$ is the adjoint representation. A spin structure on $M$ is then an equivalence class of framings.

Remark 4.1. The terminology used in mathematical literature and theoretical physics literature is somewhat different. In mathematical literature, a frame refers to a set of vectors at a given point, whereas in theoretical physics literature, a frame refers to a set of vector fields. In the current section, as well as in Sec. VII, we use terminology prevalent in mathematical literature. See also Remark 1.4.

Note that a choice of reference framing on $M$ provides a trivialization of the tangent bundle $T M$ so that any other framing is related to this reference framing by a smooth function $f: M$ $\rightarrow S^{+}(3,1)$. Two framings corresponding to functions $f_{1}$ and $f_{2}$ are equivalent in the above sense if and only if there exists a smooth function $h: M \rightarrow S L(2, \mathbb{C})$ such that $f_{2} \cdot \operatorname{Ad} h=f_{1}$ as functions $M \rightarrow S O^{+}(3,1)$.

As the traditional definition of Lorentzian spin structure, we will use the definition from Baum, ${ }^{2,3}$ see also Bichteler. ${ }^{4}$ In the special case at hand, using the trivialization of the tangent bundle $T M$ via 
the reference frame, it reads as follows. A spin structure on $M$ is an equivalence class of commutative diagrams



where $\pi$ stands for the projection onto the first factor, and the map $\Phi$ is equivariant in that $\Phi(x, g)$ $=\Phi(x, 1) \cdot$ Ad $g$ for all $x \in M$ and $g \in S L(2, \mathbb{C})$. Two diagrams as above with the vertical maps $\Phi_{1}$ and $\Phi_{2}$ are called equivalent if there is a commutative diagram

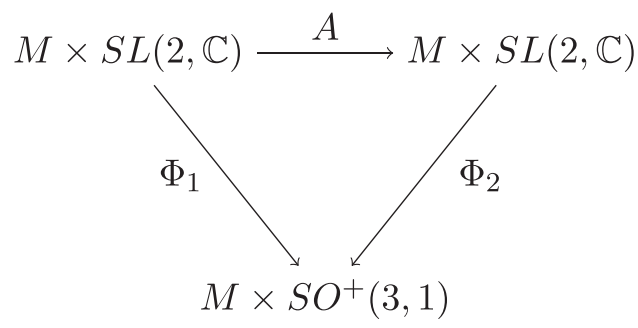

such that $\pi \circ A=\pi$ and the map $A$ is equivariant in that $A(x, g)=A(x, 1) \cdot g$ for all $x \in M$ and $g \in S L(2, \mathbb{C})$.

Theorem 4.2. For parallelizable time-orientable Lorentzian 4-manifolds, the two definitions of spin structure, our analytic definition and the traditional one, are equivalent.

Proof. Using the commutativity of the first diagram, write $\Phi(x, g)=(x, \phi(x, g))$ for some function $\phi: M \times S L(2, \mathbb{C}) \rightarrow S O^{+}(3,1)$ and observe that the equivariance condition on $\Phi$ translates into the equation $\phi(x, g)=\phi(x, 1) \cdot \operatorname{Ad} g$. Therefore, the map $\Phi$ is uniquely determined by the map $\psi: M$ $\rightarrow \mathrm{SO}^{+}(3,1)$ given by $\psi(x)=\phi(x, 1)$.

Similarly, write $A(x, g)=(x, \alpha(x, g))$ and observe that the equivariance condition on $A$ translates into the equation $\alpha(x, g)=\alpha(x, 1) \cdot g$. Therefore, the map $A$ is uniquely determined by the map $\beta: M \rightarrow S L(2, \mathbb{C})$ given by $\beta(x)=\alpha(x, 1)$. One can easily check that the second commutative diagram then simply means that $\psi_{2} \cdot \operatorname{Ad} \beta=\psi_{1}$ as functions $M \rightarrow S O^{+}(3,1)$.

This completes the proof of the equivalence of two definitions of spin structure. Note that the equivalence we established is not canonical in that it depends on the choice of reference frame.

Remark 4.3. According to (Ref. 3, Theorem 2), the equivalence classes of Lorentzian spin structures on $M$ are classified by the cohomology group $H^{1}\left(M ; \mathbb{Z}_{2}\right)$. This is an analogue of the well-known classification of Riemannian spin structures, see Proposition 7.5 and Remark 7.6.

\section{TOPOLOGICAL AND GEOMETRIC RESTRICTIONS}

In Secs. I-III, we gave an analytic definition of the concept of Lorentzian spin structure. This definition works only for parallelizable 4-manifolds equipped with time-orientable Lorentzian metric. In Sec. IV, we proved, under the assumptions of parallelizability and time-orientability, equivalence of our analytic definition to the traditional one. It is natural to examine how restrictive these assumptions are.

Proposition 5.1. A non-compact time-orientable Lorentzian 4-manifold $M$ is parallelizable if and only if it is spin. 
Proof. According to Theorem 1 of Baum, ${ }^{3}$ a time-orientable Lorentzian manifold $M$ is spin if and only if $w_{2}(M)=0$. Therefore, if $M$ is parallelizable, it is obviously spin. Conversely, the tangent bundle of $M$ is classified by a homotopy class of maps $M \rightarrow B S O^{+}(3,1)$. Since the inclusion of $S O(3)$ as a subgroup into $S^{+}(3,1)$ is a deformation retract, the classifying spaces $\mathrm{BSO}^{+}(3,1)$ and $\mathrm{BSO}(3)$ are homotopy equivalent. According to Dold-Whitney, ${ }^{7}$ the homotopy classes of maps $M \rightarrow B S O(3)$ are classified by the second Stiefel-Whitney class $w_{2}(M)$ and the first Pontryagin class $p_{1}(M) \in H^{4}(M ; \mathbb{Z})$. Since $M$ is non-compact, the group $H^{4}(M ; \mathbb{Z})$ vanishes, and the result follows.

In theoretical physics, the prevalent view is that physically meaningful spacetimes (Lorentzian 4-manifolds) are those that are non-compact and time-orientable. Thus, for physically meaningful spacetimes, our parallelizability assumption is the necessary and sufficient condition for the existence of spin structure.

One can, of course, adopt a purely mathematical approach and study Lorentzian 4-manifolds that are either compact or non-time-orientable or both. For such Lorentzian 4-manifolds, our analytic definition of spin structure may not work.

\section{CLASSIFICATION BEYOND SPIN STRUCTURE}

Let us consider all possible operators corresponding to the specified Lorentzian metric [see (2.1)], with specified charges [see (3.1) and (3.2)] and specified spin structure (see Definition 3.1). It turns out that it is possible to classify them further as follows.

Let us define the covariant subprincipal symbol $L_{\mathrm{csub}}(x)$ in accordance with formula

$$
L_{\mathrm{csub}}:=L_{\mathrm{sub}}+\frac{i}{16} g_{\alpha \beta}\left\{L_{\text {prin }}, \operatorname{adj} L_{\text {prin }}, L_{\text {prin }}\right\}_{p_{\alpha} p_{\beta}},
$$

where $\{F, G, H\}:=F_{x^{\alpha}} G H_{p_{\alpha}}-F_{p_{\alpha}} G H_{x^{\alpha}}$ is the generalised Poisson bracket on matrix-functions and adj is the operator of matrix adjugation

$$
F=\left(\begin{array}{ll}
a & b \\
c & d
\end{array}\right) \mapsto\left(\begin{array}{rr}
d & -b \\
-c & a
\end{array}\right)=: \operatorname{adj} F
$$

from elementary linear algebra. Now take an arbitrary smooth matrix-function (3.3) and consider the gauge transformation (3.4). It was shown in Ref. 9 that transformation (3.4) of our differential operator $L$ induces the transformation

$$
L_{\mathrm{csub}} \mapsto R^{*} L_{\mathrm{csub}} R
$$

of its covariant subprincipal symbol.

Comparing formulae (1.2) and (6.1), we see that the standard subprincipal symbol and covariant subprincipal symbol have the same structure, only the covariant subprincipal symbol has a second correction term designed to "take care of" special linear transformations in the vector space of unknowns $v: M \rightarrow \mathbb{C}^{2}$. The standard subprincipal symbol (1.2) is invariant under changes of local coordinates (its elements behave as scalars), whereas the covariant subprincipal symbol (6.1) retains this feature but gains an extra $S L(2, \mathbb{C})$ covariance property. In other words, the covariant subprincipal symbol (6.1) behaves "nicely" under a wider group of transformations.

Formulae (3.5) and (6.3) imply that the covariant subprincipal symbol can be uniquely represented in the form

$$
L_{\mathrm{csub}}(x)=L_{\text {prin }}(x, A(x))
$$

where $A=\left(A_{1}, A_{2}, A_{3}, A_{4}\right)$ is some real-valued co-vector field. We interpret the co-vector field appearing in formula (6.4) as the electromagnetic co-vector potential.

It is easy to see that the electromagnetic co-vector potential is invariant under gauge transformations (3.4), so it can be used for the purpose of further classification of our operators: the electromagnetic co-vector potential defines the operator uniquely modulo a transformation (3.4). Note, however, that this finer classification is not particularly interesting from the topological perspective because co-vector fields form a vector space. 


\section{THE 3-DIMENSIONAL RIEMANNIAN CASE}

Let $M$ be a connected smooth 3-manifold without boundary, not necessarily compact. As in Sec. I, let $L$ be a $2 \times 2$ formally self-adjoint non-degenerate first order linear differential operator. In dealing with the 3 -dimensional case, we make the additional assumption

$$
\operatorname{tr} L_{\text {prin }}(x, p)=0, \quad \forall(x, p) \in T^{*} M .
$$

Note that imposing condition (7.1) in the 4-dimensional setting would not make sense because it would contradict non-degeneracy (1.3).

It is easy to see that under the assumption (7.1), the non-degeneracy condition (1.3) for our $2 \times 2$ operator $L$ is equivalent to the condition

$$
\operatorname{det} L_{\text {prin }}(x, p) \neq 0, \quad \forall(x, p) \in T^{*} M \backslash\{0\} .
$$

But (7.2) is the standard ellipticity condition. Thus, in this section, we work with $2 \times 2$ formally self-adjoint elliptic first order linear differential operators $L$ with trace-free principal symbols which act over a connected smooth 3-manifold $M$ without boundary.

By analogy with Lemma 1.2, we have

Lemma 7.1. The manifold $M$ admits an elliptic operator $L$ with trace-free principal symbol if and only if it is parallelizable.

The proof of Lemma 7.1 is similar to the proof of Lemma 1.2.

Further on, we assume that our 3-manifold $M$ is parallelizable.

It is known ${ }^{13,17}$ that a 3-manifold is parallelizable if and only if it is orientable. Therefore, further on, we assume that our 3-manifold $M$ is orientable. Orientability is our only topological restriction.

We define the metric in accordance with formula (2.1). It is easy to see that this metric is Riemannian.

From now on, the Riemannian metric is assumed to be fixed. We will work with all possible $2 \times 2$ formally self-adjoint elliptic first order linear differential operators with trace-free principal symbols corresponding, in the sense of formula (2.1), to the given metric. Arguing as in Sec. II, it is easy to see that the set of such operators is non-empty.

We specify an orientation on our manifold and define the topological charge of our operator as

$$
\mathbf{c}_{\text {top }}:=-\frac{i}{2} \sqrt{\operatorname{det} g_{\alpha \beta}} \operatorname{tr}\left(\left(L_{\text {prin }}\right)_{p_{1}}\left(L_{\text {prin }}\right)_{p_{2}}\left(L_{\text {prin }}\right)_{p_{3}}\right)=\operatorname{sgn} \operatorname{det} e_{j}^{\alpha},
$$

and compare with formula (3.1). Of course, as we are now working in the 3-dimensional setting, the free indices in formula (7.3) run through the values 1, 2, 3. Further on, we assume the topological charge to be fixed.

In order to classify our operators further, we introduce an arbitrary smooth $2 \times 2$ special unitary matrix-function $R$,

$$
R: M \rightarrow S U(2),
$$

and, as in Sec. III, consider the gauge transformation (3.4). Comparing formulae (3.3) and (7.4), we see that we are now more restrictive in our choice of matrix-functions $R$ which is because we want to preserve condition (7.1)

We define spin structure in the 3-dimensional Riemannian setting in accordance with Definition 3.1, having in mind the restricted choice of operators and gauge transformations. We begin by restating our analytic definition in terms of framings. By a frame at a point $x \in M$, we mean a positively oriented orthonormal frame in the tangent space $T_{x} M$ and by a framing of $M$ a choice of a frame at every point $x \in M$ depending smoothly on the point. Framings exist because all orientable 3-manifolds $M$ are parallelizable. Furthermore, in our case, we have an explicit formula establishing a one-to-one correspondence between trace-free principal symbols and framings: this is formula (1.5) with indices $j$ and $\alpha$ restricted to the set of values 1,2, 3. Note that this one-to-one correspondence between trace-free principal symbols and framings was first observed in (Ref. 5, Appendix A). Any two framings of the same manifold $M$ are related by a uniquely defined smooth function $f: M \rightarrow S O(3)$. Rephrasing Definition 3.1 with the restricted choice of operators and gauge 
transformations, we will say that two framings are equivalent if the function $f$ relating them factors as

$$
f: M \rightarrow S U(2) \stackrel{\text { Ad }}{\longrightarrow} S O(3),
$$

where $\mathrm{Ad}: S U(2) \rightarrow S O(3)$ is the adjoint representation. A spin structure on $M$ is then an equivalence class of framings.

The following result was announced, without proof, in (Ref. 1, Sec. 7).

Theorem 7.2. For orientable Riemannian 3-manifolds, our analytic definition of spin structure is equivalent to the standard definition of (Ref. 10, Sec. 2.1).

Proof. The proof of equivalence from Theorem 4.2 goes through with little change once we replace the adjoint representation $\mathrm{Ad}: S L(2, \mathbb{C}) \rightarrow S O^{+}(3,1)$ by the adjoint representation Ad: $S U(2) \rightarrow S O(3)$.

In what follows, we provide a different proof of Theorem 7.2. This proof only works when $M$ is compact but it has the advantage that the equivalence it provides is canonical. The standard definition of spin structure we will use in this proof is the one often used by topologists; it can be found in Kaplan, ${ }^{12}$ Definition 1.7, or Milnor, ${ }^{15}$ Alternative Definition 2 (see also Remark 7.7 regarding the latter definition at the end of this section).

According to that definition, a spin structure on $M$ is a homotopy class of almost-framings of $M$. By an almost-framing of $M$, one means a framing of the punctured manifold

$$
M_{0}:=M \backslash\{\text { point }\}
$$

Two framings of $M_{0}$ are said to be homotopic if they can be connected by a path of framings of $M_{0}$. Any framing of $M$ gives rise to a homotopy class of almost-framings of $M$ by restricting it to $M_{0}$ and taking the homotopy class of this restriction.

Proposition 7.3. This gives a well-defined map $\psi$ from the set of spin-structures on $M$ as defined above to the set of the homotopy classes of almost-framings of $M$.

Proof. A function $f: M \rightarrow S O(3)$ relating two framings of $M$ restricts to a function $f_{0}: M_{0}$ $\rightarrow S O(3)$ relating their restrictions to $M_{0}$. If the function $f$ factors through $M \rightarrow S U(2)$, its restriction $f_{0}$ factors through $M_{0} \rightarrow S U(2)$. However, every function $M_{0} \rightarrow S U(2)$ is homotopic to a constant function, which in addition can be chosen to send entire $M_{0}$ to the identity element of $S U(2)$. The composition of this homotopy with the adjoint representation $S U(2) \rightarrow S O(3)$ then provides a homotopy of the induced framings of $M_{0}$.

Proposition 7.4. The map $\psi$ is a bijection from the set of spin-structures on $M$ as defined above to the set of the homotopy classes of almost-framings of $M$.

Proof. For the purposes of this proof, fix a reference framing of $M$ so that any other framing is obtained from the reference framing by applying a function $f: M \rightarrow S O(3)$. This identifies the set of all framings of $M$ with the set $\operatorname{Maps}(M, S O(3))$. The equivalence relation on the framings translates into an equivalence relation on $\operatorname{Maps}(M, S O(3))$, two functions $f, g: M \rightarrow S O(3)$ being equivalent if and only if there exists a function $h: M \rightarrow S U(2)$ such that $g(x)=\operatorname{Ad} h(x) \cdot f(x)$ for all $x \in M$. Note that the point-wise multiplication makes both $\operatorname{Maps}(M, S U(2))$ and $\operatorname{Maps}(M, S O(3))$ into groups and the induced map

$$
\operatorname{Ad}_{*}: \operatorname{Maps}(M, S U(2)) \rightarrow \operatorname{Maps}(M, S O(3))
$$

into a group homomorphism. The set of spin-structures on $M$ is then identified with the quotient $\operatorname{Maps}(M, S O(3)) / \operatorname{Im~} \operatorname{Ad}_{*}$. The latter set in general does not carry a natural group structure because $\operatorname{Im~} \mathrm{Ad}_{*}$ need not be a normal subgroup.

Next, fix a reference framing of $M_{0}$ by restricting the reference framing from $M$. The set of the homotopy classes of almost-framings of $M$ is then identified with the set $\left[M_{0}, S O(3)\right]$ of the 
homotopy classes of maps $M_{0} \rightarrow S O(3)$. With respect to this identification and the one from the previous paragraph, the map

$$
\psi: \operatorname{Maps}(M, S O(3)) / \operatorname{Im~Ad}_{*} \longrightarrow\left[M_{0}, S O(3)\right]
$$

is given by the formula $\psi(f)=\left[f_{0}\right]$, where $\left[f_{0}\right]$ stands for the homotopy class of the map $f_{0}: M_{0} \rightarrow S O(3)$ obtained by restricting $f: M \rightarrow S O(3)$ to $M_{0}$.

The map $\psi$ is surjective because any map $M_{0} \rightarrow S O(3)$ extends to a map $M \rightarrow S O(3)$ due to the fact that $\pi_{2}(S O(3))=0$. The map $\psi$ is also injective: suppose $f, g: M \rightarrow S O(3)$ are such that their restrictions $f_{0}, g_{0}: M_{0} \rightarrow S O(3)$ are homotopic. Then $g_{0} \cdot f_{0}^{-1}: M_{0} \rightarrow S O(3)$ is homotopic to the constant map taking the entire $M_{0}$ to the identity element in $S O(3)$. Therefore, $g_{0} \cdot f_{0}^{-1}$ induces a trivial homomorphism $\pi_{1}\left(M_{0}\right) \rightarrow \pi_{1}(S O(3))$ on the fundamental groups. Keeping in mind that $g_{0} \cdot f_{0}^{-1}$ is the restriction of $g \cdot f^{-1}$ and that $\pi_{1}(M)=\pi_{1}\left(M_{0}\right)$, we conclude that the map $g \cdot f^{-1}$ induces a trivial homomorphism $\pi_{1}(M) \rightarrow \pi_{1}(S O(3))$ as well. The lifting criterion applied to the double covering Ad $: S U(2) \rightarrow S O(3)$ then implies that $g \cdot f^{-1}$ lifts to a map $h: M \rightarrow S U(2)$, thereby ensuring that $g=\operatorname{Ad} h \cdot f$. This completes the proof.

Proposition 7.5. The set of spin-structures on $M$ is in a bijective correspondence with the cohomology group $H^{1}\left(M ; \mathbb{Z}_{2}\right)$.

Proof. Proposition 7.4 identifies the set of spin-structures on $M$ with the set $\left[M_{0}, S O(3)\right]$. Since $S O(3)=\mathbb{R P}^{3}$, one can use the cellular approximation theorem to identify the latter set with $\left[M_{0}, \mathbb{R} \mathrm{P}^{\infty}\right]$. Here, $\mathbb{R} \mathrm{P}^{\infty}$ is the infinite dimensional real projective space, which is known to have the homotopy type of the Eilenberg-MacLane space $K\left(\mathbb{Z}_{2}, 1\right)$. Therefore, $\left[M_{0}, \mathbb{R P}^{\infty}\right]=\left[M_{0}, K\left(\mathbb{Z}_{2}, 1\right)\right]=H^{1}\left(M_{0} ; \mathbb{Z}_{2}\right)$; see, for instance, Theorem 4.57 of Hatcher. ${ }^{11}$ To finish the proof, one simply observes that $H^{1}\left(M_{0} ; \mathbb{Z}_{2}\right)$ $=H^{1}\left(M ; \mathbb{Z}_{2}\right)$.

Remark 7.6. Proposition 7.5 is a well-known fact and it can be proved in a number of different ways. See, for instance, (Ref. 10, second Proposition on p. 40) or Ref. 14.

Remark 7.7. Milnor ${ }^{15}$ defines a spin-structure on a CW-complex $M$ as the homotopy class of framings on the 1-skeleton $M^{(1)}$ which can be extended to framings on the 2-skeleton $M^{(2)}$. The fact that this definition is equivalent to the definition of Kaplan ${ }^{12}$ for 3-manifolds $M$ follows by combining the fact that $M^{(2)}$ is a deformation retract of $M_{0}$ with the Puppe exact sequence

$$
0=\left[\bigvee S^{2}, S O(3)\right] \longrightarrow\left[M^{(2)}, S O(3)\right] \longrightarrow\left[M^{(1)}, S O(3)\right]
$$

of the cofibration $M^{(1)} \rightarrow M^{(2)} \rightarrow \bigvee S^{2}$; see, for instance, Davis-Kirk (Ref. 6, Theorem 6.42).

\section{ACKNOWLEDGMENTS}

Zhirayr Avetisyan and Dmitri Vassiliev were supported by EPSRC Grant No. EP/M000079/1. Nikolai Saveliev was supported by LMS Grant No. 21420, EPSRC Grant No. EP/M000079/1, and the Simons Collaboration Grant No. 426269.

\footnotetext{
${ }^{1}$ Avetisyan, Z., Fang, Y.-L., and Vassiliev, D., "Spectral asymptotics for first order systems," J. Spectral Theory 6, 695-715 (2016).

${ }^{2}$ Baum, H., Spin-Strukturen und Dirac-Operatoren über pseudoriemannschen Mannigfaltigkeiten (Teubner-Texte zur Mathematik, 1981)

${ }^{3}$ Baum, H., "Spinor structures and Dirac operators on pseudo-Riemannian manifolds," Bull. Pol. Acad. Sci., Math. 32, 165-171 (1985).

${ }^{4}$ Bichteler, K., "Global existence of spin structures for gravitational fields," J. Math. Phys. 9, 813-815 (1968).

${ }^{5}$ Chervova, O., Downes, R. J., and Vassiliev, D., "Spectral theoretic characterization of the massless Dirac operator," J. London Math. Soc. 89, 301-320 (2014).

${ }^{6}$ Davis, J. and Kirk, P., Lecture Notes in Algebraic Topology (American Mathematical Society, Providence, RI, 2001).

${ }^{7}$ Dold, A. and Whitney, H., "Classification of oriented sphere bundles over a 4-complex," Ann. Math. 69, 667-677 (1959).

${ }^{8}$ Downes, R. J. and Vassiliev, D., "Spectral theoretic characterization of the massless Dirac action," Mathematika 62, 701-718 (2016).
} 
${ }^{9}$ Fang, Y.-L. and Vassiliev, D., "Analysis as a source of geometry: A non-geometric representation of the Dirac equation," J. Phys. A: Math. Theor. 48, 165203 (2015).

${ }^{10}$ Friedrich, T., Dirac Operators in Riemannian Geometry, Volume 25 of Graduate Studies in Mathematics (American Mathematical Society, Providence, RI, 2000).

${ }^{11}$ Hatcher, A., Algebraic Topology (Cambridge University Press, Cambridge, 2002).

12 Kaplan, S., "Constructing framed 4-manifolds with given almost framed boundaries," Trans. Am. Math. Soc. 254, 237-263 (1979).

${ }^{13}$ Kirby, R., The Topology of 4-Manifolds, Volume 1374 of Lecture Notes in Mathematics (Springer, 1989).

${ }^{14}$ Lawson, Jr., H. B. and Michelsohn, M.-L., Spin Geometry (Princeton University Press, Princeton, New Jersey, 1989).

${ }^{15}$ Milnor, J., "Spin structures on manifolds," Enseign. Math. 9, 198-203 (1963).

${ }^{16}$ Safarov, Yu. and Vassiliev, D., The Asymptotic Distribution of Eigenvalues of Partial Differential Operators (American Mathematical Society, Providence, RI, 1997).

${ }^{17}$ Stiefel, E., "Richtungsfelder und Fernparallelismus in $n$-dimensionalen Mannigfaltigkeiten," Comment. Math. Helvetici 8 , 305-353 (1935). 